\title{
SURGERY IN GENERAL
}

\section{A quick and effective method of limb preparation with health, safety and efficiency benefits}

\author{
N Naderi', K Maw ${ }^{2}$, M Thomas $^{3}$, DE Boyce ${ }^{1}$, K Shokrollahi ${ }^{4}$ \\ ${ }^{1}$ Welsh Centre for Burns and Plastic Surgery, Swansea, UK \\ ${ }^{2}$ Swansea University, UK \\ ${ }^{3}$ Abertawe Bro Morgannwg University Health Board, Swansea, UK \\ ${ }^{4}$ Mersey Regional Plastic Surgery Unit, Whiston Hospital, Liverpool, UK
}

ABSTRACT

INTRODUCTION Pre-operative limb preparation (PLP) usually involves lifting the limb and holding it in a fixed 'static' posture for several minutes. This is hazardous to theatre staff. Furthermore, 'painting' the limb can be time consuming and difficult areas such as between toes and fingers may remain unsterile. We demonstrate the time efficiency and asepsis achieved using the 'sterile bag' preparation technique. An additional advantage is the ability to prepare and anaesthetise a limb prior to theatre, increasing efficiency substantially for units with a large throughput of cases, such as day-case hand surgery lists.

METHODS We monitored the duration of PLP in 20 patients using the 'sterile bag' technique compared to 20 patients using a conventional 'painting' method. Additionally, microbiology samples acquired from prepared upper limbs of 27 sequential patients operated on by a single surgeon over a two-month period were sent for culture immediately prior to commencement of surgery. RESULTS The mean duration of the 'sterile bag' PLP was significantly lower than that of the conventional method (24 seconds vs 85 seconds, $p=0.045)$. The technique can take as little as ten seconds $(n=1)$. Final microbiology reports showed no growth for any of the 27 patients from whom a culture sample was taken.

CONCLUSIONS The sterile bag technique is effective in achieving asepsis, has the potential to increase theatre efficiency and reduces manual handling hazards compared to the conventional method. It is now taught to all theatre staff in our hospital during manual handling training. It can be undertaken in approximately ten seconds with practice for the upper limb.

\section{KEYWORDS}

Prepare - Limb - Surgery - Bag - Sterile

Accepted 3 March 2010

CORRESPONDENCE TO

Kayvan Shokrollahi, Consultant Plastic Surgeon, Mersey Regional Burns and Plastic Surgery Service, Whiston Hospital, Warrington Road, Prescot, Merseyside L35 5DR, UKE: kshokrollahi@hotmail.com

The following paper was accepted for the Annals in March 2010 but was unfortunately not sent for copy-editing due to an administrative error. We apologise to the authors concerned for the delay in publishing their paper. Now that we have brought our submission system online we hope that such errors will be eliminated in the future.
The concept that patients can be rendered less susceptible to wound infection has been recognised for over 500 years. French surgeons Guy de Chauliac and Ambroise Paré, in the 14th and 16th century respectively, recognised that both hygiene and nutrition were important in controlling infection. In 1847 Semmelweis noted a great reduction in the incidence of sepsis among his patients when he rinsed his hands before delivering newborns. ${ }^{1}$ Koch, Pasteur and others formulated the concepts of microbes and contagion in the 19th century, proposing that infection was caused by microorganisms. In 1867 Joseph Lister published the first of his contributions, in which he describes the use of an antiseptic (carbolic acid) in the treatment of wound infections. ${ }^{2}$ As a result of these endeavours, antisepsis at the operative site was established in surgery.Throughout the years since the introduction of asepsis, a large number of agents have been used for preoperative skin preparation..$^{3-9}$ There have been extensive reports of their virtues, limitations and adverse effects. The mechanical act of skin preparation, on the other hand, has remained relatively unexplored in the literature. In the current climate of limited healthcare resources, efficiency and time management are key factors. Similarly, health and safety at work are now paramount in modern healthcare and has implications that extend into the medicolegal domain. In a previous publication, a quick and effective way for pre-operative limb preparation (PLP) was suggested..$^{10}$ Combining this method with other minor strategies can enable surgery to commence less than a minute after the patient enters the operating room. In addition, there are health and safety implications as significantly less manual handling is required. 


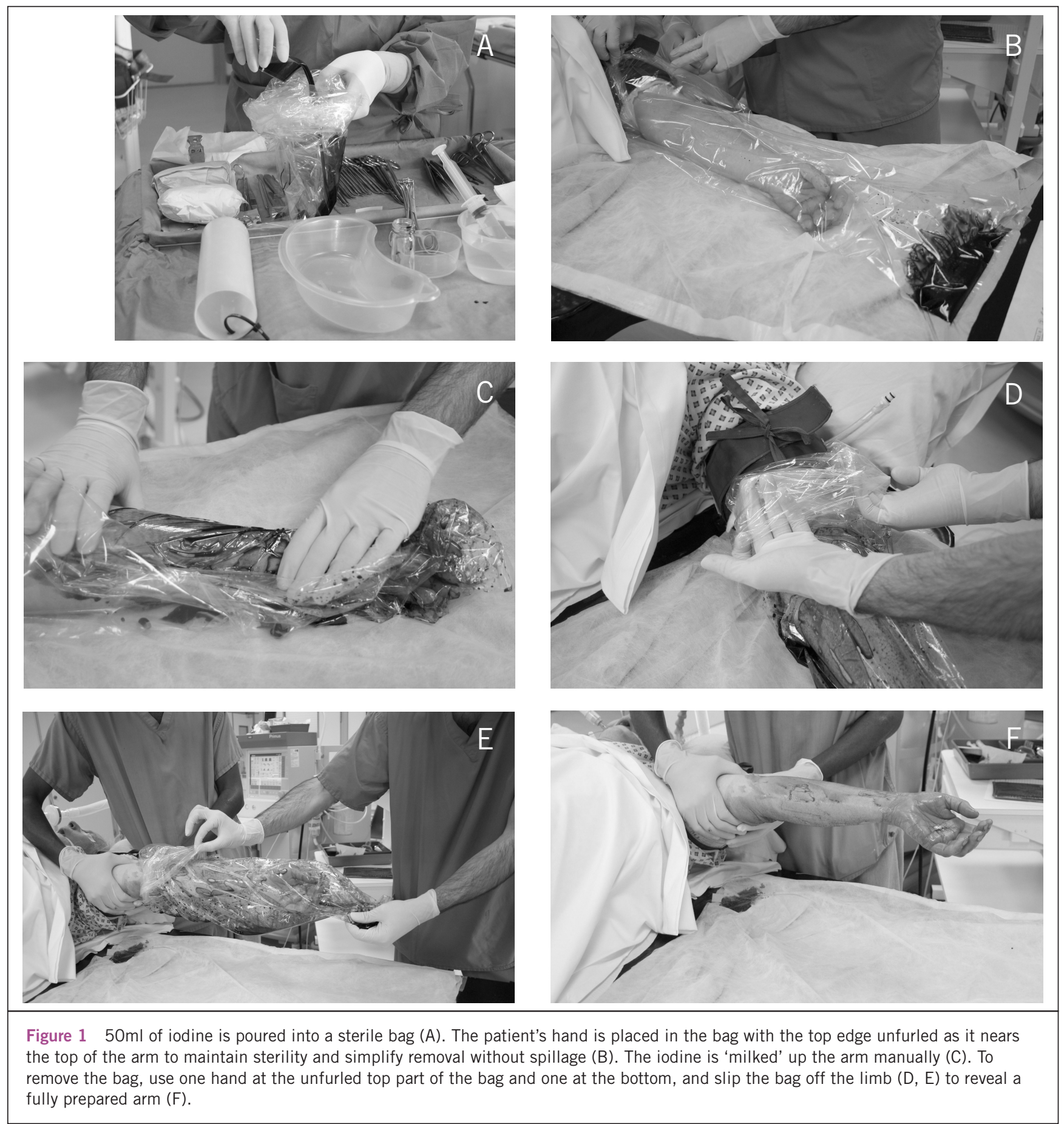

The current paper goes one step further to confirm the logical basis of this technique with evidence sufficient to support its safety microbiologically and to demonstrate timesaving relevant for safety hazard purposes and efficiency. This novel strategy aims to balance the quality of care received by patients with the safety and efficiency of work executed by operating room staff.

\section{Methods}

To prepare the arm, $50 \mathrm{ml}$ of aqueous povidone-iodine $10 \%$
(Betadine $^{\circledR}$; Seton Healthcare Group plc, Oldham, UK) is poured into a sterile bag (Multi-Aid arm bag, Frontier Multigate Ltd, Blackwood, UK; $£ 0.15$ per bag). Any sterile seethrough bag can be used and likely to be even less expensive but these are especially well suited. Paediatric sizes are also available. Alternatively, $4 \%$ chlorhexidine can be used although the non-pigment chlorhexidine solutions are less visible to those unfamiliar with or learning the technique. With practice, the senior author (KS) has used as little as $30 \mathrm{ml}$ of iodine, which is easily visible on the limb. The 
patient's hand is then placed in the bag so his or her hand rests in the solution. The iodine is then 'milked' up the arm manually.

The top part of the bag will be desterilised as it comes into contact with the top of the arm or tourniquet so, in readiness for removal of the bag and completion of PLP, the bag should be curled back on itself as it nears the top of the arm from the start. This is the area from which the bag will be removed to reveal a fully prepped arm (Fig 1). In our experience this is best achieved with one hand at the bottom of the bag and one at the curled top edge.

The time taken from the start of PLP to full preparation was monitored in 40 patients going for upper limb surgery. Half the patients were prepared using the sterile bag technique and the other half using the conventional technique. The statistical significance of the difference in the duration of PLP between the two techniques was calculated using Student's t-test. Microbiology samples, taken from the proximal aspect of the forearm prior to the start of surgery, were sent in for 27 sequential patients who had the sterile bag PLP and were operated on by a single surgeon over a two-month period.

\section{Results}

The time taken from the start of PLP to full preparation ranged from 10 to 35 seconds using the sterile bag tech-

Table 1 Preparation time in seconds using the 'sterile bag' pre-operative limb preparation (PLP) technique

\begin{tabular}{|c|c|c|}
\hline Patient & Age & PLP time \\
\hline 1 & 22 & $28 s$ \\
\hline 2 & 56 & $20 \mathrm{~s}$ \\
\hline 3 & 32 & $35 s$ \\
\hline 4 & 35 & $28 s$ \\
\hline 5 & 61 & $26 s$ \\
\hline 6 & 55 & $18 \mathrm{~s}$ \\
\hline 7 & 19 & $24 s$ \\
\hline 8 & 21 & $20 \mathrm{~s}$ \\
\hline 9 & 28 & $27 s$ \\
\hline 10 & 43 & $29 s$ \\
\hline 11 & 33 & $28 s$ \\
\hline 12 & 51 & $10 \mathrm{~s}$ \\
\hline 13 & 47 & $24 s$ \\
\hline 14 & 63 & $14 \mathrm{~s}$ \\
\hline 15 & 42 & $18 \mathrm{~s}$ \\
\hline 16 & 47 & $22 s$ \\
\hline 17 & 22 & $20 s$ \\
\hline 18 & 38 & $32 s$ \\
\hline 19 & 56 & $30 \mathrm{~s}$ \\
\hline 20 & 44 & $29 s$ \\
\hline
\end{tabular}

nique and from 71 to 97 seconds using the conventional technique. The mean duration of the sterile bag PLP was significantly lower than that of the conventional method (24. seconds vs 85 seconds, $p=0.045$ ) (Tables 1 and 2). The 27 microbiology samples, taken at the outer edges of the prepared forearms of patients undergoing the sterile bag PLP, confirmed complete asepsis in all cases.

\section{Discussion}

The concept of staff safety is sometimes overlooked when trying to ensure favourable patient outcomes. The whole theatre team is exposed to manual handling risks, including surgeons, support workers, nurses, operating department practitioners and anaesthetic staff. The UK Manual Handling Operations Regulations 1992 have placed a duty on employers to avoid hazardous manual handling operations where reasonable and practicable, to make a formal assessment of the presenting risks and to subsequently reduce the risks of injury to the lowest level achievable. Injuries to staff members have led to previous root cause analyses but without a satisfactory solution.

The conventional preparation process involves lifting the limb and holding it (often at arm's length) away from your own body in a fixed 'static' posture for several minutes. Current evidence suggests that harm is caused to the musculoskeletal system when a part of the body is held for

Table 2 Preparation time in seconds using the conventional pre-operative limb preparation (PLP) method

\begin{tabular}{|lll|}
\hline Patient & Age & PLP time \\
1 & 28 & $80 \mathrm{~s}$ \\
2 & 18 & $93 \mathrm{~s}$ \\
3 & 22 & $97 \mathrm{~s}$ \\
4 & 29 & $85 \mathrm{~s}$ \\
5 & 67 & $78 \mathrm{~s}$ \\
6 & 85 & $81 \mathrm{~s}$ \\
7 & 35 & $71 \mathrm{~s}$ \\
8 & 33 & $90 \mathrm{~s}$ \\
9 & 46 & $95 \mathrm{~s}$ \\
10 & 52 & $79 \mathrm{~s}$ \\
11 & 57 & $76 \mathrm{~s}$ \\
12 & 35 & $90 \mathrm{~s}$ \\
13 & 27 & $78 \mathrm{~s}$ \\
14 & 48 & $80 \mathrm{~s}$ \\
15 & 42 & $95 \mathrm{~s}$ \\
16 & 28 & $84 \mathrm{~s}$ \\
17 & 17 & $85 \mathrm{~s}$ \\
18 & 19 & $86 \mathrm{~s}$ \\
19 & 52 & $75 \mathrm{~s}$ \\
20 & 61 & $82 \mathrm{~s}$ \\
\hline
\end{tabular}


30 seconds or more, with the most harmful postures being forward flexion of $>30$ degrees. ${ }^{11}$ PLP using the sterile bag technique lasted $<30$ seconds in the majority of patients (Table 1) but $>70$ seconds in all patients using the conventional method (Table 2). Furthermore, the sterile bag provides a sterility barrier while the antiseptic agent is either resting in it or being milked up the limb as on the operating table. Lifting is only required briefly for the bag to be removed and the limb is ready for draping, significantly reducing manual handling hazards to theatre staff.

Sullivan et al determined the effectiveness of PLP with a clear fluid antiseptic and an iodine-based solution using the 'swab on a stick' technique over 60 and 90 seconds. ${ }^{12} \mathrm{~A}$ preparation time of 90 seconds was more effective than that of 60 seconds $(p=0.0019)$. It is our interpretation that this was because it was less likely that parts of the limb were missed out. This is therefore less relevant in relation to a limb that is completely immersed in antiseptic solution. The fact that visibly staining solutions were more effective than invisible solutions in Sullivan et al's study also lends weight to this theory.

The sterile bag technique can be applied to the lower limb as well, reducing the amount of time required to lift the leg for PLP. Moreover, the bag can be unfurled to the level of the foot to allow the leg to be lifted by non-sterile personnel while surgical drapes are applied although the fluid is best first drained from the bag through a small hole.

Theatre efficiency is an important concept. It can lead to a greater throughput of patients, meaning less waiting time and possibly improved outcomes, especially in the context of trauma. When using the sterile bag PLP, the following additional procedures could potentially enable surgery to start within a minute of the patient entering theatre:

1. a surgical team of at least two individuals;

2. insertion of local anesthetic on the ward 10-20 minutes before surgery, eg as the previous case is being closed/ dressed;

3. a limb tourniquet applied (when necessary) but not inflated; and

4. a limb can be placed in a sterile bag with antiseptic solution and secured at the top of the limb (especially the arm) either with an elastic band across the top of the tourniquet or with tape prior to entering theatre.

When the patient enters theatre, the arm can be simultaneously exsanguinated and prepared using an exsanguinator, the tourniquet inflated, the sterile bag removed and a single-piece drape applied in less than 60 seconds.

A final major advantage of PLP taking place on the ward while the patient is awake is that there is no manual handling required as the patient can elevate his or her own limbs.

In $2008,2,854$ operations were performed on the upper limb in our plastic surgery theatres alone. Our study has shown an average reduction in PLP time of 61 seconds when using the sterile bag technique instead of the conventional method. This could potentially lead to an additional 48 hours of available theatre time a year. Furthermore, on a weekly basis there would be a 65 -minute reduction in limb lifting time by theatre staff. This is a significant reduced annual lifting hazard for staff, efficiency aside, and could equate to a great decrease in the likelihood of injury as a result of static overload. We developed a schematic, including simple images of the new procedure, in poster form and a short training video that is available on all theatre computers for staff training and reference. New training material is now used on corporate central manual handling training for all theatre staff.

\section{Conclusions}

Those individuals for whom this article is most relevant, especially those who have ever attempted to force a 'swab on a stick' between a patient's toes, will immediately realise that complete skin coverage with antiseptic solution is logically achieved better with fingers and toes resting initially in a puddle of solution in a small enclosed space. Our study has shown a 'safe' level of aseptic efficacy in addition to other benefits, providing an evidence base for this method over and above its logical simplicity. As with any technique, there is a short learning curve and spillages may occur until the technique has been used a few times. We would therefore not recommend preparing a lower limb with this method until individuals are familiar with preparing the upper limbs as the learning curve with the former is steeper.

The strategy for PLP described in our paper demonstrates how patient safety, theatre efficiency, and health and safety of staff can simultaneously be improved. It can lead to increased speed and turnover as well as a reduction in manual handling risks to staff by greatly reducing the need to lift and support limbs. If there is willing and strategic implementation, junior staff and nurses can anaesthetise and prepare a limb on the ward prior to surgery and have a tourniquet in place ready for inflation, reducing the time a precious theatre operating table is used for anything other than actual surgery.

\section{References}

1. Sinclair W. Semmelweiss: His Life and His Doctrine. Manchester: Manchester University Press; 1909.

2. Lister J. On the antiseptic principle in the practice of surgery. Br Med J 1867; 2: 246-248.

3. Ghosh J, Maisels DO, Woodcock AS. Preoperative skin disinfection. Br J Surg 1967; 54: 551-553

4. Lowbury EJ, Lilly HA. Use of 4 per cent chlorhexidine detergent solution (Hibiscrub) and other methods of skin disinfection. Br Med J 1973; 1: 510-515.

5. Cruse PJ, Foord R. A five-year prospective study of 23,649 surgical wounds. Arch Surg 1973; 107: 206-210.

6. Rodeheaver G, Bellamy W, Kody M et al. Bactericidal activity and toxicity of iodine-containing solutions in wounds. Arch Surg 1982; 117: 181-186.

7. Sebben JE. Surgical antiseptics. J Am Acad Dermatol 1983; 9: 759-765.

8. Ayliffe GA, Noy MF, Babb JR et al. A comparison of pre-operative bathing with chlorhexidine-detergent and non-medicated soap in the prevention of wound infection. J Hosp Infect 1983; 4: 237-244.

9. Hayek LJ, Emerson JM. Preoperative whole body disinfection - a controlled clinical study. J Hosp Infect 1988; 11 Suppl B: 15-19.

10. Shokrollahi K, Kirwadi A, Hiew L, Laing H. Preparing for upper and lower limb surgery in seconds: a technique with implications for theatre efficiency and manual handling. J Plast Reconstr Aesthet Surg 2008; 61: 1,123-1,124.

11. Miedema MC, Douwes M, Dul J. Recommended maximum holding times for prevention of discomfort of static standing postures. Int J Ind Ergon 1997; 19 9-18.

12. Sullivan PJ, Healy CE, Hirpara KM et al. An assessment of skin preparation in upper limb surgery. J Hand Surg Eur Vol 2008; 33: 513-514. 